\title{
Evolution des Rapports de Genre dans les Sociétés Bamiléké et Bëti du Cameroun
}

\author{
Hélène Kamdem Kamgno \\ Institut de Formation et de Recherche Démographiques, \\ IFORD, B.P. 1556 Yaoundé, Cameroun ${ }^{1}$ \\ hekamgno@yahoo.fr
}

\section{Résumé}

Cet article examine l'importance de la dimension culturelle des rapports de genre pour la compréhension des comportements démographiques. Nous utilisons des données quantitatives et qualitatives sur deux groupes ethniques au Cameroun (les Bamiléké et les Bëti) dans une analyse comparative des opinions vis-à-vis des statuts et des rôles masculins et féminins. Comparés au modèle culturel traditionnel de base, ces analyses permettent d'évaluer l'évolution des modèles culturels relatifs au genre. Elles permettent ensuite d'examiner l'influence de certaines caractéristiques individuelles des conjoints. Les résultats montrent d'importants changements au sein de chaque groupe ethnique, liés en partie à l'instruction et l'urbanisation. Toutefois, l'influence de ces facteurs se fait beaucoup plus sentir en société Bëti qu'en société Bamiléké, reflet d'une mutation du traditionnel vers le modernisme différente entre les deux ethnies.

Mots clés : Genre, ethnies, éducation, urbanisation, Cameroun

\section{Abstract}

This article examines the importance of cultural constructions of gender and their impact on demographic behaviour. We combine qualitative and quantitative data on two ethnic groups in Cameroon (the Bëti and Bamiléké) to compare individual perceptions about men and women's positions and roles within these two ethnic groups. Such analyses help assess the historical changes in gender norms. The analyses also explore the influences of various partner characteristics. Results show substantial changes that stem in part from rising education and urbanisation. However, these factors are more influential among the Bëti than the Bamiléké, a reflection of differences in the transition from tradition to modernity between the two ethnic groups.

Keywords : Gender, education, ethnics, urbanisation, Cameroon

\section{Introduction}

Nombre d'études antérieures ont montré l'influence du statut des femmes africaines sur leur comportement démographique (Boserup 1970, 1985;
Mason 1984; Safilios-Rothschild 1985; Oppong 1988). Toutefois, le statut de la femme dans ces études était considéré comme «un état prédéterminé alors qu'en réalité, [il résulte] d'une construc-

I. Tél. (237) 22232947 / 99993853 / 220048 I I 
tion sociale et d'un partage de rôles sociaux entre les sexes masculin et féminin» (Farges et Perrot, 1993 cités par Locoh et al., 1996). Ces études couvrent la dimension socioéconomique du "statut de la femme» appréhendé à travers l'instruction et l'activité économique. Elles occultent ainsi d'autres dimensions telles que l'activité domestique (Oppong 1988 ; Boserup 1970). Bien plus, elles ignorent l'importance des constructions socioculturelles des rôles et rapports entre hommes et femmes au sein de la famille, la société, et le lieu de travail (Locoh et Labourie-Racapé, 1999).

Cette étude s'appuie sur deux groupes ethniques du Cameroun (les Bamiléké et les Bëti) pour examiner l'importance de la dimension culturelle des rapports de genre dans la compréhension des comportements démographiques. L'article examine la formation et l'évolution des rapports de genre au sein des deux groupes, ainsi que l'influence de ces rapports sur les comportements démographiques. Notre hypothèse principale est que les rapports entre conjoints sont culturellement modulés, et sont soit largement assignés (société Bamiléké) soit largement acquis (société Bëti). Néanmoins, ces modèles traditionnels s'effritent sous l'influence de l'instruction et de l'urbanisation. A terme, les femmes à statut acquis développent une plus grande propension au partage du pouvoir domestique et à la prise de décision, notamment dans la gestion des revenus et la planification familiale. Ces hypothèses sont testées avec des données quantitatives et qualitatives.

L'article est structuré en trois sections. La première définit les concepts centraux de l'étude, notamment les rapports de genre et les statuts assignés et acquis. La deuxième section analyse et compare les opinions individuelles sur les statuts et rôles des hommes et femmes, et l'évolution historique de ces rôles. La troisième section analyse les rapports de genre au sein du couple, selon les caractéristiques individuelles des conjoints, notamment le niveau d'instruction, l'activité économique, le type d'union, et l'écart d'âges entre conjoints.

\section{Cadrage conceptuel}

Les rapports de genre désignent l'ensemble des statuts socialement conférés aux hommes et aux femmes. Ils déterminent leurs rôles dans la société ou dans la famille et peuvent s'appréhender aussi bien au niveau collectif qu'individuel. Par rapport au groupe social de référence, les rôles individuels doivent être conformes aux normes et valeurs préétablies. Les représentations individuelles des statuts et des rôles des hommes et des femmes traduisent ainsi les manifestations inconscientes des rapports de genre prescrits par le groupe. Ces représentations peuvent être étudiées en recueillant les opinions individuelles sur les statuts et rôles sociaux assignés aux hommes et femmes ou alors en examinant directement les comportements des individus par rapport à ces rôles et statuts. Sur la base de ces opinions et comportements, on peut distinguer deux types de statuts : les statuts assignés et les statuts acquis. Les statuts assignés sont conférés à chaque sexe par les us et coutumes de l'ethnie. Ils sont intimement liés à la tradition et constituent une manifestation tradition- 
nelle des modèles culturels. Les statuts acquis résultent des expériences accumulées (socialisation, éducation, activités professionnelles, ...) en tant qu'acteur socioéconomique et ils découlent des efforts individuels à rompre avec la division traditionnelle des rôles.

Les recherches démographiques utilisant l'approche genre restent peu nombreuses mais elles montrent que cette perspective peut éclairer la compréhension de plusieurs phénomènes tels que la mortalité et la santé, la nuptialité, la migration, la constitution de la famille et du ménage, la fécondité et la planification familiale (Antonella Pinnelli 1997; Locoh et Labourie-Racapé 1997; Maffioli et Ajello 1997). En ce qui concerne la fécondité, plusieurs études montrent que la pratique de la contraception varie en fonction des attitudes individuelles mais aussi en fonction des rapports entre conjoints (Andro et Hertrich 2000; Dodoo 1995; Ezeh 199I; Philips et al., 1997; Kouye 1997; Ngoy 1993; Noumbissi et Sanderson 1996). De manière empirique, le type de statut peut s'évaluer en examinant la division sexuelle des tâches domestiques et agricoles, la division sexuelle en matière de prise de décision, les différences d'âges entre conjoints, le type d'union, l'instruction ou la formation différentielle offerte aux garçons et aux filles, les activités professionnelles ou les actions sociales des hommes et des femmes.

\section{Background theorique et revue de litterature}

La littérature sur notre sujet distingue le "sexe», qui regroupe tout ce qui relève du biologique, du "genre» qui a trait non seulement à la différence physique, mais aussi à la différenciation sociale et culturelle des sexes, avec un accent particulier sur leur hiérarchisation et la différenciation des fonctions qui en découlent. Les études sur le genre rendent ainsi compte des différences de rôles entre hommes et des femmes dans la société ou la famille ainsi que des inégalités des rapports qu'ils $y$ entretiennent. En dehors de leur but scientifique, ces études poursuivent aussi un objectif éthique/politique dans la mesure où elles visent à promouvoir un partage équitable des rôles et des rapports entre hommes et femmes. Sur le plan politique, l'on est passé de l'approche «femme et développement» ou approche "du bien être» qui, dans les année 50 et 60 , considérait les femmes comme un groupe vulnérable, pauvre et passif, qui devaient recevoir de l'assistance de la communauté ou d'organismes de protection sociale «welfare approach» à l'approche «intégration des femmes au développement» (Kabré et al., 1999). Cette dernière approche, qui a soutenu des actions ponctuelles auprès des femmes (par ou pour les femmes), s'est avérée insuffisante car elle a souvent renforcé leur marginalisation en les confinant à des domaines traditionnellement féminins ou en ignorant le rôle capital des hommes comme détenteurs du pouvoir et du contrôle des biens (CEE, 1995, cité par Locoh et al., 1996). Sous l'égide des trois dernières Conférences Mondiales organisées par les Nations Unies (Caire 1994, Pékin 1995 et Copenhague 1996), l'approche "genre et développement» se substitue à l'approche «intégration des femmes au développement». L'approche "genre et 
développement» se veut transversale à l'ensemble des politiques, programmes et projets de développement, tout en maintenant des interventions ciblées sur les femmes pour pallier les inégalités récurrentes entre les sexes. Les promoteurs de ce courant ont démontré qu'on ne peut espérer améliorer la condition de la femme et promouvoir l'égalité entre les sexes que si l'on remet en cause les rapports de pouvoir historiquement et socialement valorisés entre les hommes et les femmes. On ne peut pas s'adresser isolément aux femmes en tant que groupe-cible sans intégrer le système qui régit les relations de ce groupe avec les autres dans la collectivité (Kabré et al., 1999).

\section{Donnees et methodes}

Pour tester nos hypothèses, nous combinons des données quantitatives et qualitatives. Les premières proviennent de l'Enquête Démographique et de Santé réalisée au Cameroun en 1998 (EDSC 98). Elles décrivent les rapports de genre effectivement vécus dans la discussion et la prise de décision au sein du couple, ainsi que les caractéristiques individuelles des conjoints telles que l'instruction, l'activité économique, l'écart d'âges, le type d'union. Les données qualitatives sont issues de I'Enquête Culture, Relations de Genre, Comportements Sexuels et MST/SIDA au Cameroun (ECRGCS), réalisée par I'IFORD en 2000 dans le cadre de ses activités de recherche. Ces données qualitatives issues des discussions de groupe et des entretiens individuels renseignent sur les normes et valeurs socioculturelles qui régissent les rapports de genre. Elles décrivent les opinions des participants en matière de mariage, de prise de décision au sein du couple, de scolarisation, d'activités agricoles et professionnelles, de participation aux tâches domestiques et d'actions sociales. Ces deux données sont complétées par une revue de littérature sur les deux contextes socioculturels traditionnels.

Pour les données qualitatives, nous avons analysé le contenu des discussions de groupes et des entretiens individuels réalisés dans l'ECRGCS afin de comparer les opinions sur les rôles et pouvoirs des hommes et des femmes au sein de chacun des deux groupes ethniques et, par conséquent, leurs normes et valeurs socioculturelles relatives au genre. Pour les données relatives aux opinions, aux caractéristiques individuelles, à la discussion et la prise de décision au sein du couple nous avons utilisé des modèles descriptifs univariés et bivariés.

\section{Resultats: opinions sur les statuts et roles des hommes et femmes}

\section{Mariage et sexualité}

\section{Importance du mariage}

Des entretiens individuels et des discussions de groupes, il ressort que l'on attend prioritairement d'une fille le mariage et la procréation alors que c'est d'abord la réussite matérielle, le mariage et la procréation qui sont normativement attendus du garçon. Ces attentes varient au sein des deux sociétés. Aussi bien chez les Bamiléké que chez les Bëti, certains informateurs reléguaient le mariage et la procréation à un niveau secondaire et d'autres ne les évoquaient même pas parmi les attentes des parents vis-à-vis de leurs 
filles. Au contraire, ces parents attendent de leur fille «qu'elle grandisse et que dans l'avenir, qu'elle fasse quelque chose qui peut les aider (...), qu'elle travaille pour les aider» (femme Bëti adulte mariée, niveau secondaire); «qu'elle devienne quelqu'un demain, donc qu'elle fréquente, travaille et apporte quelque chose comme l'argent» (jeune femme bamiléké mariée, niveau secondaire). Pour certains, la réussite de la fille dans la vie active semble désormais primer sur le mariage et la procréation. Alors que chez les Bamiléké, l'impact de l'instruction semble se manifester aussi bien chez les hommes que chez les femmes, chez les Bëti, il est surtout visible chez les femmes. En effet, quel que soit le niveau d'instruction, les hommes Bëti attendent d'abord de leur fille le mariage et la procréation : " elle se marie automatiquement ... " (homme Bëti adulte marié, niveau primaire) ; «lorsque la fille est mise au monde, elle est appelée à aller en mariage, à aller créer une autre famille» (jeune homme Bëti marié, niveau secondaire). Même avec un niveau d'instruction élevé, certains hommes Bëti justifient le mariage comme principale attente de leur fille par la dot «on va m'apporter la dot (...) on peut devenir riche» (homme Bëti adulte marié, niveau secondaire».

Dans chaque groupe ethnique, le mariage est considéré comme le cadre idéal pour la procréation. Néanmoins, chez les Bëti, certains sont favorables à la fécondité pré-maritale alors qu'elle est très mal perçue chez les Bamiléké. Selon la plupart de nos informateurs Bëti, la fécondité pré-maritale peut être une bonne chose et les enfants naturels sont bien accueillis: "C'est une chance même d'avoir une fille qui a eu un enfant avant le mariage; c'est une preuve de maturité avant le mariage; mon petit-fils qui est même là est né comme ça» (leader traditionnel Bëti, 64 ans) ; « ma fille me donne un enfant avant le mariage (...) je serai tellement content si j'ai un remplaçant, un petit fils, cela ne peut pas amener des problèmes " (leader traditionnel Bëti, 5I ans). Chez les Bëti aussi, la fécondité pré-maritale est toujours perçue comme une preuve de fertilité chez la fille comme le prouve ce propos d'un leader traditionnel Bëti de 5I ans: «moi, je vais tolérer que ma fille me laisse un enfant avant d'aller en mariage (...) parce que si elle accouche chez moi avant d'aller en mariage, je pourrai dire que ma fille n'a pas l' "akyae " 2 ". Dans la société Bëti, la tolérance vis-àvis de la sexualité et de la fécondité prémaritale chez la jeune va en accord avec le proverbe bëti qui dit que «la fille n'est pas la femme de son père» («ngon esiki ngal esia»).

Chez les Bamiléké par contre, la fécondité pré-maritale pour une jeune fille est très mal vue car elle entraîne une perte d'estime et réduit les chances de trouver un mari. Les expressions suivantes en témoignent : «mais traditionnellement on sait qu'elle a perdu le mariage car en fait, elle n'a plus cas la même estime que celle qui part en mariage étant vierge; c'est à partir de sa virginité qu'elle a la confiance de son mari» (leader traditionnel Bamiléké, 62 ans); “ selon la tradition bamiléké, il ne faut même pas mettre l'accent du côté du garçon, c'est du côté de la fille que ça pèse (...) puisque lorsqu'elle fait un enfant avant de se marier, elle réduit ses chances d'avoir un mari » (leader tradi-

2. Une forme de stérilité due à un envoûtement. 
tionnel bamiléké, 57 ans). Dans la société bamiléké, les jeunes filles mères sont ainsi victimes d'une certaine stigmatisation. La préférence pour les parents d'envoyer la jeune fille en mariage plutôt que de la scolariser permet, entre autres, de réduire les risques liés à l'activité sexuelle avant le mariage.

\section{Fidélité}

La fidélité est un autre sujet important. Chez les Bëti, la tolérance vis-à-vis des escapades sexuelles de la jeune fille continue jusque dans le mariage. Selon nos informateurs, l'infidélité est fréquente en société Bëti. On y note même l'utilisation coutumière de ces escapades comme objet d'échange ou d'hospitalité: «moi j'entends même que quelqu'un qui a des femmes peut les passer à ses frères ou à ses visiteurs, mais dans ce cas, avant de partir, tu dois d'abord aller travailler en brousse pour le mari» (leader traditionnel Bëti, 5I ans). Nos informateurs notent aussi l'existence, au sein de la société bëti, des pratiques similaires notamment celle du «nga» et celle l'«eban» ${ }^{3}$. Malgré cette tolérance, l'infidélité reste source de conflit. En particulier, celle de la femme est condamnée, même si elle n'est pas punie sévèrement. "Quand on découvre qu'une femme mariée est infidèle, la première des choses est qu'on la ramène à la raison $(. .$.$) ; il existe des assises$ familiales pendant lesquelles on essaie toujours d'arranger un peu " (leader traditionnel Bëti, 56 ans ); "on appelle le mari et la femme et on demande à celleci de choisir si elle veut continuer sa vie dans le mariage ou avec l'homme adultérin " (leader traditionnel Bëti, 64 ans). A l'homme qui a commis l'adultère avec la femme, on exige parfois une amende, à remettre à l'homme victime. Cette amende peut être constituée d'une "dame jeanne " de vin rouge, un coq ou bien même un mouton (leader traditionnel Bëti, 5I ans). Dans la société Bamiléké, contrairement au pays Bëti, selon les avis de nos informateurs, l'infidélité féminine est peu fréquente, voire rare. "La femme infidèle (...), c'est très rare " (leader traditionnel Bamiléké, 52 ans). L'infidélité y est condamnée et sévèrement punie. "Chez les Bamiléké, celle qui est infidèle, on l'appelle bordelle (...), les punitions sont toujours très sévères " (leader traditionnel bamiléké, 52 ans). "On peut la mettre sur la place publique et la huer" (leader traditionnel bamiléké, 57 ans), "ou l'expulser du village" (leader traditionnel bamiléké, 62 ans). L'infidélité de l'homme est aussi mal perçue et sanctionnée, mais avec une sévérité moindre par rapport à celle de la femme car l'homme bamiléké est par essence polygame: "chez les Bamiléké, un homme peut avoir chez lui même dix femmes mais une femme ne peut pas avoir dix hommes (...), une femme avec un homme, mais un homme peut avoir plusieurs femmes " (leader traditionnel bamiléké, 52 ans) ; "parce qu'on dit qu'un homme peut être capable d'avoir même cinquante femmes» (discussion de groupe leaders traditionnel bamiléké). La polygamie est ainsi acceptée et même recommandée chez

3. Le principe du «nga» consiste à ce qu'un homme sexuellement impuissant ou économiquement pauvre donne sa femme ou l'une de ses femmes --avec le consentement de cette dernière-- à un autre homme. L'«eban» quant à lui consiste à ce qu'un homme mette en gage sa femme soit pour rembourser une dette contractée, soit pour payer des amendes exigées lors d'un procès perdu. 
les Bamiléké.

\section{Polygamie}

Les opinions sur la polygamie varient selon l'ethnie et le sexe. Chez les Bëti, hommes et femmes désapprouvent la polygamie. Selon eux, elle engendre de nombreux conflits liés à la cohabitation de plusieurs coépouses. Elle est source d'infidélité des femmes à cause de leur insatisfaction sexuelle et matérielle. Chez certains hommes, au lieu de la juguler, la polygamie favorise plutôt leur infidélité. La polygamie peut cependant se justifier lorsqu'elle survient en cas de contrainte, notamment lorsque la première épouse n'a pas eu d'enfants. Et, dans ce cas, les avis sont partagés quant au nombre de femmes qu'un homme polygame doit avoir. Certains de nos informateurs pensent que le nombre de femmes d'un polygame dépend de ses moyens alors que d'autres pensent que ce nombre doit se limiter à un maximum de deux (selon la majorité des femmes) ou trois (selon les leaders traditionnels). Chez les Bamiléké, les opinions sont partagées. Toutes les femmes et certains hommes estiment que la polygamie engendre de nombreux conflits. A l'instar des Bëti, ceux qui désapprouvent la polygamie estiment qu'elle favorise l'infidélité féminine à cause de l'insatisfaction sexuelle et matérielle. Les leaders traditionnels et certains hommes quant à eux estiment que la polygamie est une bonne chose lorsqu'elle est bien gérée. En effet, selon les leaders traditionnels, une polygamie bien gérée confère à l'homme des titres de notabilité et, par conséquent renforce sa dignité.

Ils soulignent toutefois qu'elle est difficile à gérer, raison pour laquelle sa gestion garantit des titres de notabilité.
Les propos suivants sont significatifs à cet égard :

"La polygamie ne paie pas, mais on peut supporter; elle ne paie pas en ce sens que, celle-ci est malade, il faut intervenir, l'enfant est malade à gauche, à droite, il faut intervenir, il y a beaucoup d'intervention, mais on est obligé de supporter» (leader traditionnel Bamiléké, 56 ans, polygame de 5 épouses avec 18 enfants). Abondant dans le même sens, un autre leader traditionnel Bamiléké déclare «moi, j'ai des problèmes, moi en tant que marié de plusieurs femmes, j'ai beaucoup de problèmes. II y a des deuils que je fais, il y a des enfants que je dois nourrir, je ne peux plus penser à être riche. Tout ce que je travaille moi-même personnellement, je ne fais que m'occuper des enfants et de leurs mères "(leader traditionnel Bamiléké, 52 ans, polygame de 8 épouses, au moins 42 enfants). Ainsi, même ceux qui approuvent la polygamie reconnaissent aussi que la polygamie engendre non seulement de nombreux conflits et de nombreuses charges, mais aussi des cas d'infidélité qui peuvent survenir à cause du mauvais encadrement des femmes tant sexuellement que matériellement. Les opinions sur le nombre maximum d'épouses sont variées. Ceux qui désapprouvent la polygamie situent ce nombre à un maximum de trois. Les autres pensent que le nombre dépend des moyens financiers et peut s'élever jusqu'à dix épouses.

\section{Ecart d'âges entre conjoints}

Chez les Bëti, la plupart des informateurs masculins désapprouvent un grand écart d'âges. Chez les femmes, une minorité pense que les femmes qui sont beaucoup plus jeunes que leurs maris tendent à mépriser ces derniers, 
et précarisent ainsi leurs mariages (leaders traditionnels Bëti). Certaines femmes estiment que ce genre de mariage est un moyen de domination de l'homme sur la femme et que cette dernière reçoit de lui un "vieux sang ". Toutefois, la majorité des femmes et une minorité d'hommes pensent que l'écart d'âges entre conjoints ne pose pas de véritables problèmes. "L'amour n'a pas d'âges et chacun a son goût; ce qui compte c'est la sincérité de l'amour" (femmes leaders d'associations Bëti). Certaines femmes estiment par ailleurs que les vieux ne dérangent pas autant que les jeunes et ils encadrent bien leurs épouses.

Chez les Bamiléké en revanche, toutes nos informatrices et la plupart des hommes estiment qu'un grand écart d'âges entre conjoints n'est pas une bonne chose. Selon les femmes, leurs consoeurs qui sont dans de tels mariages vieillissent vite; elles ont peur de leurs maris et ne seront jamais heureuses. Dans le même ordre d'idée, les hommes estiment que dans un foyer de ce genre, la femme sera constamment dominée par l'homme car "elle $a$ un esprit très faible par rapport à celui du mari." Bien que minoritaires, certains hommes pensent qu'un grand écart d'âges entre conjoints n'a aucun inconvénient. Toutes ethnies et sexes confondus, nos informateurs soutiennent que les jeunes filles qui acceptent ce genre de mariage sont simplement matérialistes et attirées par les richesses des hommes plus vieux. Dans ce genre de mariage l'infidélité des femmes est fréquente à cause de l'insatisfaction sexuelle auprès de vieux maris. Les résultats montrent aussi que les informateurs de niveau secondaire expriment le plus souvent des opinions défavorables à la polygamie et à un grand écart d'âges entre conjoints. Ceci suggère un effet d'acculturation à travers l'instruction.

\subsection{Opinions sur les décisions du couple}

\section{Gestion des revenus}

Même si la norme d'un contrôle masculin de gestion reste vivace, les mentalités évoluent: Près de 3 et 4 personnes sur 10 respectivement chez les Bamiléké et les Bëti prônent le partage du pouvoir de gestion entre l'homme et la femme (Tableau I.I). Cette proportion est plus faible chez les Bamiléké que chez les Bëti, suggérant une plus grande adhésion des populations Bëti à la participation féminine à la prise de décision économique au niveau domestique. Par ailleurs, indépendamment de l'ethnie, les femmes sont de loin plus susceptibles de préférer une gestion féminine ou conjointe. Une gestion plus commune est jugée " plus fructueuse " (discussions de groupes hommes mariés adultes Bëti) ou "facilite la vie au sein du couple" (discussion de groupe femme mariées adultes Bamiléké).

S'agissant spécifiquement des revenus de la femme, plus de la moitié des enquêtés - indépendamment du sexe et de l'ethnie - pensent que l'homme ne doit pas gérer le revenu du travail de son épouse (Tableau I). Cette décision devrait revenir soit exclusivement à la femme, soit aux deux conjoints. L'appropriation exclusive de ce pouvoir de décision par la femme est surtout recommandée lorsqu'elle a commencé à travailler avant de se marier car le mariage ne signifie pas «la perte de toute autonomie pour une femme, les 
choses acquises avant restent siennes" (discussions de groupes jeunes hommes mariés Bamiléké) ou "si elle s'est mariée sous un régime de biens séparés "(discussions de groupes femmes célibataires et jeunes hommes mariés, Bamiléké ; discussions de groupes chefs traditionnels, hommes mariés et femmes mariées de Ngallan, Bëti). On note une fois de plus que les Bëti sont plus favorables que les Bamiléké au partage de ce pouvoir entre l'homme et la femme (3l \% contre $24 \%$ ).

Quelle que soit l'ethnie, par rapport aux résultats observés précédemment au niveau de la prise de décision sur la répartition du revenu du ménage, l'idée d'une prise de décision exclusive par la femme sur l'utilisation du revenu de son travail est plus fréquente. Une décision exclusive de la part de la femme serait donc plus tolérée lorsqu'il s'agit du fruit de son propre travail que lorsqu'il s'agit du revenu total du ménage. Par ailleurs, dans chaque groupe ethnique, les hommes sont plus favorables que les femmes à la détention de ce pouvoir par l'homme ou à son partage entre les deux conjoints tandis que les femmes le sont plus pour la détention de ce pouvoir par la femme. Les écarts hommesfemmes sont toutefois plus importants chez les Bamiléké que chez les Bëti.

Tableau I Opinions sur la décision en matière de gestion économique selon l'ethnie et le sexe (ECRGCS, 2000)

\begin{tabular}{llll}
\hline Opinions sur la décision en matière & \multicolumn{2}{c}{ Bamiléke } & \multicolumn{2}{c}{ Bêti } \\
\cline { 2 - 4 } de gestion économique & Hommes Femmes Ens & Hommes Femmes & Ens
\end{tabular}

Prise de décision sur l'utilisation du (*) revenu du ménage $\left(^{*}\right)$

(*)

\begin{tabular}{lllllll}
\hline L'homme & 63,2 & 52,2 & 57,8 & 50,8 & 33,4 & 42,3 \\
La femme & 8,7 & 17,4 & 13,0 & 14,9 & 26,9 & 20,8 \\
L'homme et la femme & 28,1 & 30,4 & 29,2 & 31,9 & 39,4 & 35,5 \\
(Effectif) & $(367)$ & $(362)$ & $(729)$ & $(323)$ & $(308)$ & $(631)$ \\
\hline $\begin{array}{l}\text { Prise de décision sur l'utilisation du } \\
\text { revenu du travail de la femme (*) }\end{array}$ & & & $(*)$ & & \\
\hline L'homme & 42,0 & 32,9 & 37,4 & 35,0 & 30,8 & 33,0 \\
La femme & 28,3 & 34,8 & 31,6 & 27,6 & 38,0 & 32,6 \\
$\begin{array}{l}\text { L'homme et la femme } \\
\text { L'homme si biens communs }\end{array}$ & 28,1 & 20,2 & 24,2 & 33,7 & 27,7 & 30,7 \\
La femme si bien séparés & 1,1 & 8,8 & 4,9 & 2,8 & 2,9 & 2,9 \\
\hline (Effectif) & 0,0 & 3,0 & 1,5 & 0,0 & 0,3 & 0,2 \\
\hline Notes: & $(367)$ & $(362)$ & $(729)$ & $(323)$ & $(308)$ & $(631)$ \\
\hline
\end{tabular}

Notes: $\left(^{*}\right)$ : khi2 significative au seuil de $5 \%$; (-): khi2 non significative au seuil de $5 \%$; devant les intitulés des variables (I ère colonne), on a le seuil par rapport à l'ethnie alors que dans les colonnes 2 et 5 , il s'agit des seuils par rapport au sexe dans chacune des ethnies ; dans chaque colonne, le complément par rapport à $100 \%$ du cumul concerne la modalité " autres » ou « NSP », pour tenir compte des contraintes liées aux effectifs, le khi2 a été calculé en omettant cette dernière modalité. 


\subsection{Décision en matière de fécondité}

Indépendamment de l'ethnie, les enquêtés estiment que les décisions en matière de contraception devraient revenir aux femmes (environ 6 personnes sur dix dans chaque ethnie, tableau 2). Chez les Bamiléké, hommes et femmes ont quasiment les mêmes opinions. Chez les Bëti par contre, les femmes sont plus favorables que les hommes à la détention de ce pouvoir par la femme. Citant les attentes d'une femme en matière de procréation, une femme mariée Bëti déclare : « ... enfin, c'est bien la femme qui doit veiller sur son cycle menstruel, notamment les périodes fécondes et infécondes afin de planifier et d'organiser la procréation au sein du couple».

Tableau 2 Opinions sur la décision en matière de fécondité selon l'ethnie et le sexe (ECRGCS, 2000)

\begin{tabular}{lllllll}
\hline $\begin{array}{l}\text { Opinions sur la décision en } \\
\text { matière de fécondité }\end{array}$ & \multicolumn{3}{c}{ Bamiléke } & & Bêti \\
\cline { 2 - 7 } $\begin{array}{l}\text { Prise de décision sur la } \\
\text { pratique contraceptive au } \\
\text { sein du couple (-) }\end{array}$ & $(-)$ & Femmes & Ens & Hommes & Femmes & Ens \\
\cline { 2 - 7 } & & & & $(*)$ & & \\
\hline L'homme & 19,1 & 21,4 & 20,2 & 21,8 & 9,7 & 15,9 \\
La femme & 59,8 & 60,8 & 60,3 & 57,6 & 68,2 & 62,8 \\
L'homme et la femme & 20,8 & 16,1 & 18,5 & 19,0 & 18,2 & 18,6 \\
(Effectif) & $(366)$ & $(360)$ & $(726)$ & $(321)$ & $(308)$ & $(629)$ \\
\hline Prise de décision sur le & $(*)$ & & & $(*)$ & & \\
nombre d'enfants & & & & & & \\
\hline dans un couple $\left.{ }^{*}\right)$ & 48,5 & 39,5 & 44,0 & 57,0 & 44,5 & 50,9 \\
L'homme & 9,8 & 23,5 & 16,6 & 9,9 & 16,2 & 13,0 \\
La femme & 40,3 & 35,4 & 37,9 & 29,1 & 37,7 & 33,3 \\
L'homme et la femme & $(367)$ & $(362)$ & $(729)$ & $(323)$ & $(308)$ & $(631)$ \\
\hline (Effectif) & & & & & & \\
\hline
\end{tabular}

Notes : idem Tableau I.

S'agissant ensuite du nombre d'enfants, les individus pensent que ce pouvoir appartient beaucoup plus à l'homme (Tableau 2). Toutefois, une proportion relativement importante d'individus estime que cette décision devrait être prise de commun accord (38\% et 33 $\%$ respectivement chez les Bamiléké et chez les Bëti). Ceci suggère une évolution des mœurs et une prise de conscience de la nécessité de dialogue entre hommes et femmes à ce sujet. Par rapport au résultat sur la prise de décision en matière de contraception (qui favorise le pouvoir féminin), il peut paraître paradoxal que la décision sur le nombre d'enfants soit davantage confiée aux hommes. Le paradoxe est seulement apparent: l'homme déciderait si oui ou non faire un enfant, et il reviendrait alors à la femme de mettre en œuvre les moyens nécessaires pour accomplir ces vœux. Contrairement à ce qui s'observe pour les autres décisions, les Bamiléké sont plus favorables que les Bëti au partage de ce pouvoir entre conjoints (Tableau 2). Indépendamment de l'ethnie, les femmes sont plus favorables que les hommes à une féminisation de ce pouvoir. Par ailleurs, alors que les hommes Bamiléké sont plus favorables que les femmes au 
partage de ce pouvoir entre les deux plus que des hommes. époux, chez les Bëti, les femmes le sont

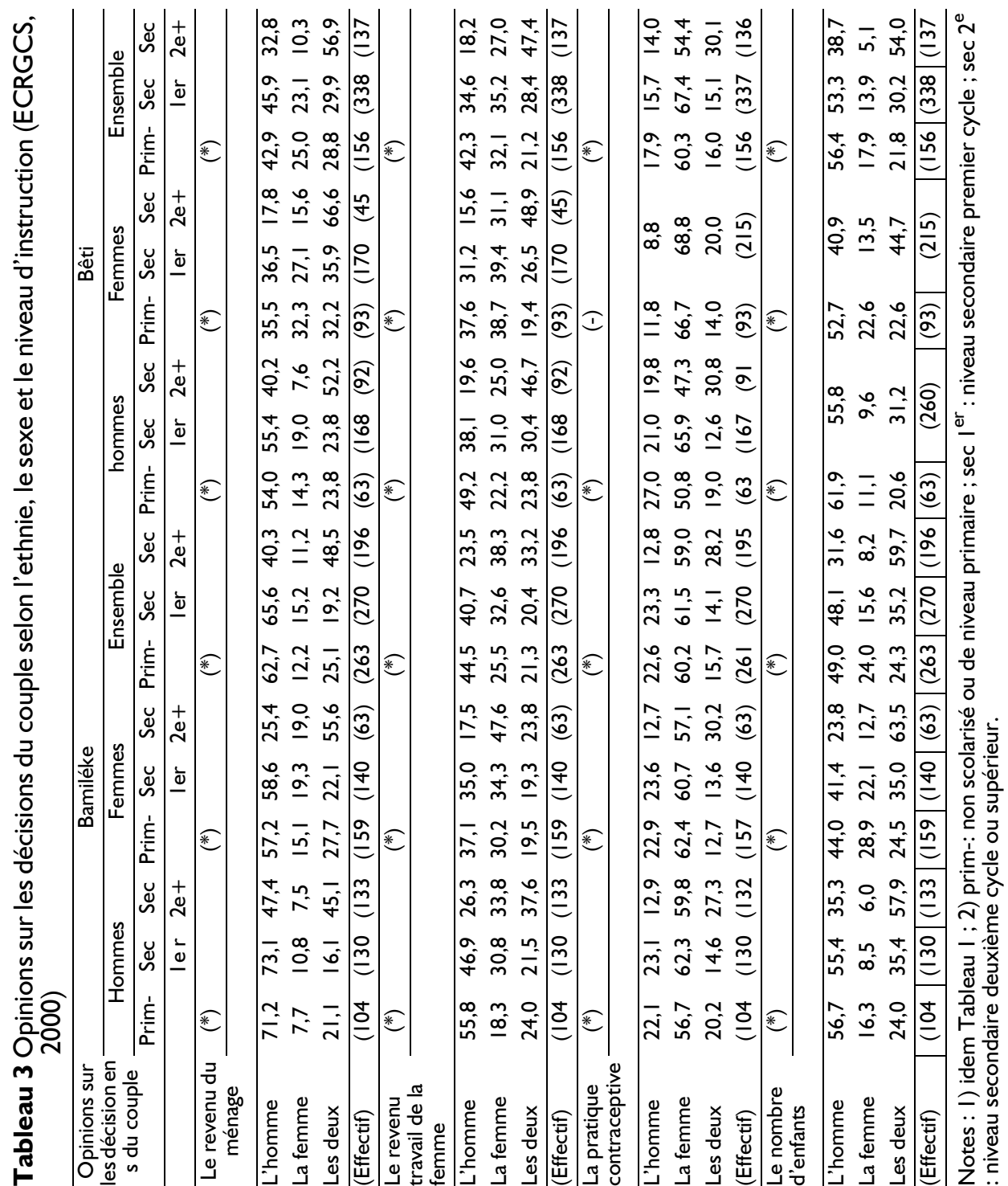

Une analyse des effets nets de l'éducation indique que le pourcentage des individus favorables au partage du pouvoir de décision augmente avec le niveau d'instruction (Tableau 3). L'instruction semble ainsi être un facteur d'acculturation favorisant la participation conjointe à la prise des décisions au sein du couple. En général, les écarts sont plus grands chez les Bëti que chez les Bamiléké, plus traditionalistes.

Les deux ethnies diffèrent dans leur opinion sur une gestion exclusivement féminine du revenu de la femme et de la pratique contraceptive. Chez les Bamiléké, l'on observe une association 
positive entre niveau d'instruction et disposition favorable à une prise décision exclusive par les femmes. Chez les Bëti au contraire, cette association est négative, surtout chez les femmes. De même chez les Bëti, alors que l'associa- tion entre instruction et acceptation du pouvoir féminin en matière de pratique contraceptive est négative chez les hommes, chez les Bamiléké, le niveau d'instruction n'a pas d'effet.

Tableau 4 Opinions sur les décisions du couple selon l'ethnie, le sexe et le milieu de résidence (ECRGCS, 2000)

\begin{tabular}{|c|c|c|c|c|c|c|c|c|c|c|c|c|}
\hline & \multicolumn{6}{|c|}{ Bamiléke } & \multicolumn{6}{|c|}{ Bêti } \\
\hline & \multicolumn{2}{|c|}{ Hommes } & \multicolumn{2}{|c|}{ Femmes } & \multicolumn{2}{|c|}{ Ensemble } & \multicolumn{2}{|c|}{ Hommes } & \multicolumn{2}{|c|}{ Femmes } & \multicolumn{2}{|c|}{ Ensemble } \\
\hline & Urb & Rur & Urb & Rur & Urb & Rur & Urb & Rur & Urb & Rur & Urb & Rur \\
\hline $\begin{array}{l}\text { Décision sur l'utilisation } \\
\text { du revenu du ménage }\end{array}$ & $(-)$ & & $(-)$ & & $(-)$ & & $(*)$ & & (*) & & $(*)$ & \\
\hline L'homme & 60,9 & 65,6 & 50,3 & 54,0 & 55,7 & 59,7 & 63,7 & 41,5 & 45,6 & 25,1 & 55,0 & 33,4 \\
\hline La femme & 6,5 & 10,9 & 21,4 & 13,8 & 13,7 & 12,3 & 9,6 & 18,6 & 24,8 & 28,5 & 16,9 & 23,5 \\
\hline L'homme et la & 32,6 & 23,5 & 28,3 & 32,2 & 30,6 & 28,0 & 25,2 & 36,7 & 29,6 & 45,9 & 27,3 & 41,2 \\
\hline (Effectif) & $(184)$ & $(183)$ & $(173)$ & $(189)$ & (357) & (372) & $(135)$ & $(188)$ & $(125)$ & $(183)$ & $(260)$ & (37I) \\
\hline $\begin{array}{l}\text { Décision sur l'utilisation } \\
\text { du revenu du travail de } \\
\text { la femme }\end{array}$ & $(*)$ & & $(-)$ & & $(*)$ & & $(-)$ & & $(-)$ & & $(-)$ & \\
\hline L'homme & 35,3 & 48,6 & 30,6 & 34,9 & 33,1 & $4 I, 7$ & 40,0 & 31,4 & 35,2 & 27,9 & 37,7 & 29,6 \\
\hline La femme & 35,9 & 20,8 & 42,2 & 28,0 & 38,9 & 24,4 & 29,7 & 26,1 & 38,4 & 37,8 & 33,8 & 31,8 \\
\hline L'homme & 28,3 & 27,9 & 22,0 & 18,5 & 25,1 & 23,1 & 28,1 & 37,8 & 26,4 & 28,4 & 27,3 & 33,2 \\
\hline (Effectif) & $(184)$ & $(183)$ & $(173)$ & (189) & (357) & (372) & $(135)$ & 188 & $(125)$ & $(183)$ & $(260)$ & $(37 I)$ \\
\hline $\begin{array}{l}\text { Décision sur la pratique } \\
\text { contraceptive au sein du } \\
\text { couple }\end{array}$ & $(-)$ & & $(-)$ & & $(-)$ & & $(*)$ & & $(*)$ & & (*) & \\
\hline L'homme & 18,5 & 19,8 & 19,7 & 23,0 & 19,0 & 21,4 & 16,3 & 25,8 & 13,6 & 7,1 & 15,0 & 16,5 \\
\hline La femme & 61,4 & 58,2 & 63,6 & 58,3 & 62,5 & 58,3 & 65,2 & 52,2 & 75,2 & 63,4 & 70,0 & 57,7 \\
\hline L'homme et & 20,1 & 21,5 & 16,2 & 16,0 & 18,2 & 18,7 & 17,8 & 19,9 & 10,4 & 23,5 & 14,2 & 21,7 \\
\hline Autres (Effectif) & $(184)$ & $(182)$ & $(173)$ & $(187)$ & $(357)$ & (369) & $(135)$ & $(186)$ & $(125)$ & $(183)$ & $(260)$ & (369) \\
\hline $\begin{array}{l}\text { Décision sur l } \\
\text { d'enfants dans }\end{array}$ & $(-)$ & & $(*)$ & & $(*)$ & & $(-)$ & & $(-)$ & & $(-)$ & \\
\hline$\overline{\text { L'hor }}$ & & & & & & & & & & & & \\
\hline La femme & 43,5 & 53,6 & 39,3 & 39,7 & 41,5 & 46,5 & 53,3 & 59,6 & 41,6 & 46,5 & 47,7 & 53,1 \\
\hline L'homme et la femme & 9,8 & 9,8 & 17,9 & 28,6 & 13,7 & 19,4 & 9,6 & 10,1 & 15,2 & 16,9 & 12,3 & 13,5 \\
\hline (Effectif) & 45,1 & 35,5 & 41,6 & 29,6 & 43,4 & 32,5 & 34,1 & 25,5 & $4 I, 6$ & 35,0 & 37,7 & 30,2 \\
\hline
\end{tabular}

Notes : I) idem Tableau I ; 2$)$ urb = urbain, rul = rural.

Le Tableau 4 montre une association positive entre résidence urbaine et acceptation d'une participation conjointe à la prise de décision sur le nombre d'enfants. Chez les Bamiléké, la résidence urbaine est positivement liée à l'acceptation d'une gestion exclusive- ment féminine du revenu des femmes. L'appropriation du pouvoir de décision, même sur le revenu du travail de la femme, est plus ancrée chez les ruraux que les urbains. Chez les Bëti, contrairement à nos attentes, la résidence urbaine réduit la participation conjointe 
à la gestion des revenus de ménage et de la contraception.

\subsection{Participation masculine aux activités domestiques}

Dans chaque ethnie, beaucoup (39\% chez les Bamiléké et 33\% chez les Bëti, Tableau 5) pensent toujours que l'homme ne devrait pas participer aux activités domestiques. Chez les Bëti par exemple, le fait pour un homme d'entrer à la cuisine est une honte, une façon de se rabaisser car sa femme va le traiter de gourmand. "Moi je pense c'est la coutume de l'homme africain, de l'homme Bene. Dans notre langue, on appelle cela «ati» (noblesse), puisque vous ne pouvez pas dire à un homme Bene d'aller souffler le feu dans la cuisine ou bien d'aller éplucher les bananes ou aller faire ceci, jamais, à cause de l' "ati», parce que là, la femme va dire que mon mari là, il est «fam oloa» (homme gourmand), ce qui sera une grande honte pour lui " (leader traditionnel Bëti, 5l ans). Ainsi, pour l'homme Bëti, entrer à la cuisine lui ferait perdre sa noblesse. De même, chez les Bamiléké, certains n'imaginent pas qu'un homme puisse entrer dans la cuisine pour faire à manger, c'est humiliant «je me dis que le travail réservé à la femme, par exemple, l'homme ne peut pas aller à la cuisine préparer pour que les gens mangent en laissant la femme. La femme est là pourquoi?» (leaders traditionnels Bamiléké, 56 ans). II est important de signaler que, selon les discours de nos informateurs, aussi bien chez les Bamiléké que chez les Bëti, s'il y a une activité liée directement à la cuisine que l'homme doit faire, c'est la recherche et la fente du bois. Cela se justifie par le fait que l'homme est jugé physiquement plus fort que la femme.

\begin{tabular}{|c|c|c|c|c|c|c|}
\hline \multirow{3}{*}{$\begin{array}{l}\text { Opinions sur la } \\
\text { participation des } \\
\text { hommes aux } \\
\text { activités domestiques }\end{array}$} & \multirow{2}{*}{\multicolumn{3}{|c|}{ Bamiléke }} & \multirow{2}{*}{\multicolumn{3}{|c|}{ Bêti }} \\
\hline & & & & & & \\
\hline & Hommes & Femmes & Ens & Hommes & Femmes & Ens \\
\hline Un homme ne devrait & $(*)$ & & & $(*)$ & & \\
\hline $\begin{array}{l}\text { pas participer aux } \\
\text { activités domestiques }(*)\end{array}$ & & & & & & \\
\hline Oui & 34,3 & 42,8 & 38,5 & 26,9 & 39,1 & 32,8 \\
\hline Non & 65,7 & 56,1 & $6 I, 0$ & 72,8 & 60,3 & 66,7 \\
\hline (Effectif) & $(367)$ & $(362)$ & $(729)$ & (323) & (307) & $(630)$ \\
\hline
\end{tabular}

La proportion d'individus opposés à la participation masculine aux activités domestiques est plus élevée chez les Bamiléké que chez les Bëti, suggérant un plus grand attachement des premiers aux traditions. Curieusement, les femmes sont plus favorables que les hommes à la nonparticipation masculine aux activités domestiques. On peut penser que les femmes sont elles mêmes fières de leur place au foyer ou alors «'habitude étant une seconde nature», elles trouvent cet arrangement normal. En dépit de cet attachement à la tradition, plus de la moitié des enquêtés pensent que l'homme doit 
également participer aux travaux ménagers, surtout lorsque la femme travaille en dehors du foyer, participe aux charges du ménage, ou voyage. Le changement social à l'extérieur des foyers induirait ainsi le changement à l'intérieur. Le niveau d'instruction n'a pas d'effet significatif chez les Bamiléké, mais chez les Beti, il favorise la partici- pation masculine aux activités domestiques (Tableau 6).

La résidence urbaine est, chez les Bëti, négativement associée au refus de participation masculine aux activités domestiques, alors que chez les Bamiléké, cette relation est plutôt positive (Tableau 7$)^{4}$.

Tableau 6 Opinions sur la participation des hommes aux activités domestiques selon l'ethnie, le sexe et le niveau d'instruction (ECRGCS, 2000)

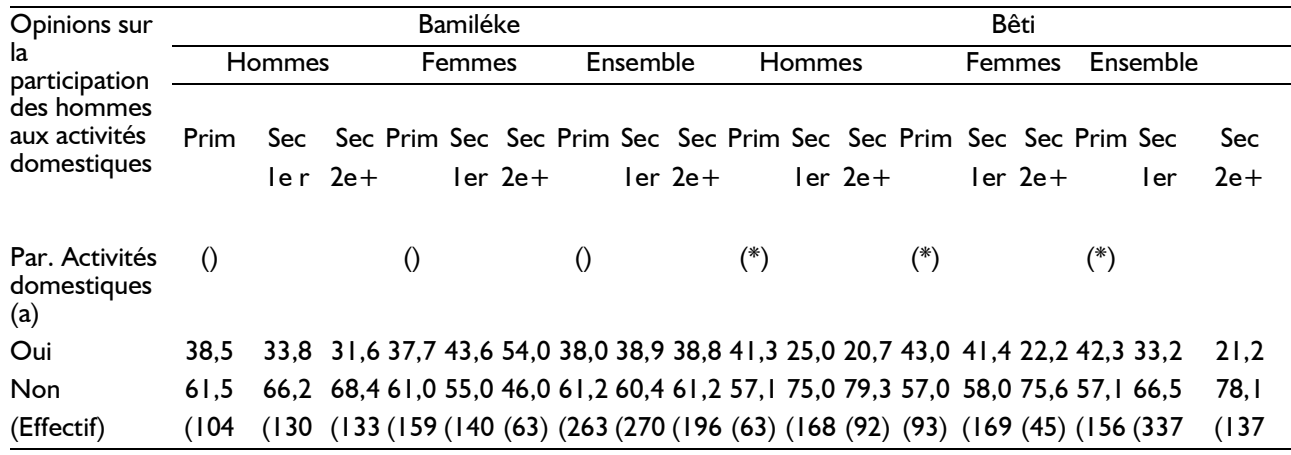

Notes : I) idem Tableau I ; 2) idem Tableau 3 ; 3) (a) = un homme devrait participer aux activités domestiques

\subsection{Opinions en matière de scolarisation}

Lorsqu'on se réfère aux discours des leaders traditionnels qui reflètent ici le modèle traditionnel de base, les résultats suggèrent que chez les Bëti, l'instruction - bien que n'étant pas mentionnée comme principal élément est citée (après le mariage et la procréation) parmi les attentes des parents vis-à-vis de leurs filles. " $\mathrm{Ce}$ qu'on attend d'une fille dans l'ethnie Beti-Ewondo, c'est qu'elle se marie et qu'elle fasse des

4. Lorsqu'on contrôle le milieu de résidence par le niveau d'instruction, la situation observée chez les Bëti se maintient quel que soit le niveau d'instruction, l'écart urbain-rural se creusant davantage au fur et à mesure que le niveau d'instruction augmente. Chez les Bamiléké, la tendance observée se maintient chez les individus de niveau primaire ou moins et chez ceux de niveau secondaire premier cycle. Ce n'est qu'à partir du niveau secondaire deuxième cycle qu'il existe une relation négative entre le fait de résider en milieu urbain et le fait de penser que l'homme ne devrait pas participer aux activités domestiques. En effet, chez les Bamiléké de niveau secondaire deuxième cycle ou plus, $37 \%$ de la population urbaine contre $42 \%$ en milieu rural pensent que les activités domestiques ne sont pas du ressort masculin. Ainsi chez les Bamiléké, l'exclusion de l'homme de la sphère domestique est si ancrée dans les mœurs que c'est à partir du niveau secondaire deuxième cycle que le fait de résider en ville réduirait la probabilité d'admettre que l'homme ne devrait pas participer aux activités domestiques. 
enfants et qu'elle soit dans l'intégrité dans son foyer, qu'elle soit digne, qu'elle fréquente au même titre que le garçon, qu'elle entre dans le développement du pays, il s'agit d'entreprendre des activités génératrices des revenus comme les champs communautaires, la fabrication du savon, ... » (femme leader traditionnel Bëti). "En premier lieu, ce sont ses études, si elle peut faire des études, sinon le mariage ... » (leader traditionnel Bëti,
5 I ans). En société Bëti, l'instruction de la fille est d'autant plus importante que chez les leaders traditionnels, l'instruction de la femme est évoquée parmi les actions qu'ils souhaiteraient que le Gouvernement entreprenne pour améliorer la situation des femmes car cela leur garantirait un emploi rémunérateur. L'instruction est ainsi bien perçue comme un moyen qui permet d'avoir une activité rémunératrice.

Tableau 7 Opinions sur la participation des hommes aux activités domestiques selon l'ethnie, le sexe et le milieu de résidence (ECRGCS, 2000)

\begin{tabular}{|c|c|c|c|c|c|c|c|c|c|c|c|c|}
\hline \multirow{3}{*}{$\begin{array}{l}\text { Opinions sur la } \\
\text { participation } \\
\text { des hommes } \\
\text { aux activités } \\
\text { domestiques }\end{array}$} & \multicolumn{6}{|c|}{ Bamiléke } & \multicolumn{6}{|c|}{ Bêti } \\
\hline & \multicolumn{2}{|c|}{ Hommes } & \multicolumn{2}{|c|}{ Femmes } & \multicolumn{2}{|c|}{ Ensemble } & \multicolumn{2}{|c|}{ Hommes } & \multicolumn{2}{|c|}{ Femmes } & \multicolumn{2}{|c|}{ Ensemble } \\
\hline & Urb & Rur & Urb & Rur & Urb & Rur & Urb & Rur & Urb & Rur & Urb & Rur \\
\hline $\begin{array}{l}\text { Un homme ne } \\
\text { devrait pas } \\
\text { participer aux } \\
\text { activités } \\
\text { domestiques }\end{array}$ & (*) & & $(*)$ & & $\left({ }^{*}\right)$ & & (*) & & $(*)$ & & $(*)$ & \\
\hline
\end{tabular}

\begin{tabular}{lcccccccccccc}
\hline Oui & 38,6 & 30,1 & 48,6 & 37,6 & 43,4 & 33,9 & 17,0 & 34,0 & 28,8 & 46,2 & 22,7 & 40,0 \\
Non & 61,4 & 69,9 & 50,3 & 61,4 & 56,0 & 65,6 & 83,0 & 65,5 & 71,2 & 52,7 & 77,3 & 59,2 \\
\cline { 2 - 10 } (Effectif) & $(184)$ & $(183)$ & $(173)$ & $(189)$ & $(357)$ & $(372)$ & $(135)$ & $(188)$ & $(125)$ & $(182)$ & $(260)$ & $(370)$ \\
\hline
\end{tabular}

Notes: I) idem Tableau I 2) idem Tableau 4

Chez les leaders traditionnels Bamiléké par contre, la scolarisation n'a pas été citée parmi les attentes des parents visà-vis de leurs filles. Dans la société traditionnelle bamiléké, on y attendait principalement des filles le mariage et la procréation. "Quand on donnait naissance à une fille, on n'attendait d'elle que le mariage à partir de 16 ans " (leader traditionnel Bamiléké, 52 ans); " on attendait qu'elle produise les semblables de son père " (leader traditionnel bamiléké, 56 ans).

Quant au garçon, aussi bien dans la société traditionnelle bamiléké que dans celle bëti, on attendait d'abord du garçon la réussite dans la vie qui est ici synonyme de l'exercice d'une activité économique et de la construction d'une maison. Dès qu'il peut supporter les charges d'une famille, l'homme peut alors se marier et procréer.

«II fallait donc seulement montrer au garçon comment effectuer les travaux du village, défricher les champs, planter le cacao, ou lui apprendre un petit métier comme la maçonnerie et la menuiserie » (leader traditionnel bëti, 76 ans). «... II doit attacher la barrière, construire la maison, c'était une question de tradition" (discussion de groupe leaders traditionnels Bamiléké). On développait pour cela aux enfants de sexe masculin des capacités nécessaires pour l'acquisition des moyens qui leur permettront de supporter ces charges dans leur vie future.

L'instruction étant bien perçue 
comme un moyen d'accès à l'emploi, cette différence entre les attentes des parents vis-à-vis des filles et garçons pourrait expliquer en partie la sousscolarisation des filles par rapport aux garçons. Dans les deux groupes, l'analyse des opinions sur la sousscolarisation des filles révèle d'importants changements par rapport au modèle traditionnel de base. En effet, la plupart de nos informateurs désapprouvent la sous-scolarisation des filles. Chez les Bëti, l'instruction n'est plus seulement perçue comme une garantie d'un bon emploi rémunérateur, mais aussi comme un facteur qui «rehausse l'image, l'estime, bref le statut de la femme" (discussion de groupe femmes Bëti), un facteur «fondamental d'émancipation de la femme" (discussion de groupe hommes Bëti). Chez les Bamiléké, la sous-scolarisation des filles est déplorable car «elle favorise la prostitution et la marginalisation des filles» (femmes Bamiléké). Quelle que soit l'ethnie, les femmes de niveau d'études secondaires, contrairement à leurs consoeurs non instruites ou de niveau primaire, attendent principalement de leur fille l'instruction ou la scolarisation, l'exercice d'un emploi rémunéré et un soutien à la famille. Les propos suivants en sont révélateurs : «qu’elle soit lettrée, quoi (...), une femme instruite afin d'être en mesure de subvenir à ses besoins" (femmes Bëti adulte mariée, niveau secondaire) ; «qu'elle puisse grandir et que dans l'avenir, qu'elle fasse quelque chose qui peut les aider (...), qu'elle travaille pour les aider " (femme mariée Bëti, niveau secondaire) ; " un enfant sage, intelligent et qu'elle progresse dans les études » (jeune femme marié Bamiléké, niveau secondaire) ; "la scolarisation, l'emploi professionnel, l'assistance à la famille et aux parents " (femme Bamiléké adulte mariée, niveau secondaire).

De même chez les hommes bamiléké, la plupart des informateurs qui ont mentionné principalement la scolarisation ou l'exercice des activités rémunératrices parmi leurs attentes à l'égard de leur fille ont un niveau d'instruction élevé : " qu'elle réussisse à l'école, travaille et gagne suffisamment d'argent afin de pouvoir aider ses parents " (homme adulte bamiléké marié, niveau secondaire). Les hommes Bëti par contre, quel que soit le niveau d'instruction, comme signalé plus haut, attendent d'abord de leur fille le mariage et la procréation.

\subsection{Analyse différentielle de la discussion et de la décision en matière de fécondité}

Quelle que soit l'ethnie, les couples homogame de niveau d'instruction élevé ou hypogame de niveau d'instruction moyen sont les plus enclins à discuter de la planification familiale (Tableau Al en annexe) $)^{5}$. Ce résultat suggère qu'au sein de ces deux groupes, l'influence de l'instruction de la femme est plus grande que celle de l'homme. Par ailleurs, le fait que le couple soit homogame de niveau d'instruc-

5. Homogame élevé = les deux conjoints ont un niveau secondaire ou plus ; Hypogame moyen = la femme a un niveau secondaire ou plus et l'homme est non scolarisé ou a un niveau primaire ; Hypergame moyen = l'homme a un niveau secondaire ou plus et la femme est non scolarisée ou a un niveau primaire; Homogame faible = les deux conjoints sont non scolarisés ou ont un niveau primaire 
tion élevé (surtout chez les Bamiléké) et le fait que le couple soit homogame de niveau d'instruction faible (surtout chez les Bëti), sont associés positivement au partage du pouvoir de décision au sein du couple sur la pratique contraceptive entre les conjoints (tableau Al en annexe). Toutefois, chez les Bamiléké, plus les conjoints ont des niveaux d'instruction différents plus la femme aurait tendance à s'approprier le pouvoir de décision sur la contraception et moins les deux conjoints participeraient ensemble à cette décision alors que chez les Bëti, plus les deux conjoints ont des niveaux d'instructions différents, plus l'homme aurait tendance à s'approprier le pouvoir de décision sur la pratique contraceptive et moins les deux participeraient ensemble à cette décision (tableau $\mathrm{Al}$ en annexe).

Le pouvoir économique est aussi important. Le fait que les deux conjoints aient le même pouvoir économique élevé et, dans une moindre mesure, le fait qu'au moins un des conjoints ait un pouvoir économique élevé, semblent favoriser la discussion de la planification familiale au sein du couple 6 . En revanche, le fait que les deux conjoints aient le même pouvoir économique faible est un facteur défavorable (Tableau A2 en annexe). Des effets similaires sont observés en matière de contraception. Le fait que les deux conjoints aient le même pouvoir économique élevé favorise la participa- tion conjointe à la prise de décision sur la pratique contraceptive au détriment de la détention de ce pouvoir par la femme (chez les Bamiléké) ou par l'homme (chez les Bëti) (Tableau A2 en annexe).

L'implication des hommes aux activités domestiques semble aussi favoriser la discussion de la planification familiale (Tableau A3 en annexe). Cela traduirait un rapprochement des conjoints qui trouveraient là un moment de discuter d'autres sujets. Chez les Bamiléké, la participation de l'homme aux activités domestiques augmente, au sein du couple, le partage du pouvoir de décision sur la pratique contraceptive entre conjoints au détriment de son appropriation par la femme. Chez les Bëti, par contre, la participation de l'homme aux activités domestiques au sein du couple réduit le partage du pouvoir de décision sur la pratique contraceptive entre conjoints au profit de sa détention par la femme.

Quelle que soit l'ethnie, surtout chez les Bamiléké, la réduction de l'écart d'âges entre conjoints favorise la discussion sein du couple sur la planification familiale (Tableau A4 en annexe). L'écart d'âges entre conjoints n'a pas un effet significatif sur la décision du couple en matière de pratique contraceptive. Lorsqu'on contrôle l'écart d'âges entre conjoints par l'instruction du couple, les différences restent toujours non significatives, à l'exception, chez les Bamiléké, des couples hypogames de

6. Homogame élevé = les deux conjoints ont le même pouvoir économique élevé (c'està dire exercent dans le commerce, les professions libérales ou sont cadres supérieurs ou techniciens des administrations publique ou privée) ; homogame faible $=$ les deux conjoints ont le même pouvoir économique faible (c'est à dire sont agriculteurs, manœuvres ou exercent dans les activités autres) ; hypogame moyen $=$ la femme a un pouvoir économique supérieur à celui de l'homme; hypergame moyen =l'homme a un pouvoir économique supérieur à celui de la femme. 
niveau d'instruction moyen. Chez ces derniers, la réduction de l'écart d'âges entre conjoints augmente au sein du couple le taux de détention par la femme du pouvoir de décision sur la pratique contraceptive au détriment de sa détention par l'homme.

Aussi bien chez les Bamiléké que chez les Bëti, le taux de discussion sur la planification familiale est plus élevé chez les couples monogames que chez les couples polygames. Le fait d'être en union monogamique constituerait ainsi, dans chacun des deux groupes ethniques, un facteur favorable à la discussion au sein du couple. De même, dans chaque groupe ethnique, davantage chez les Bamiléké, la monogamie apparaît comme un facteur qui favoriserait au sein du couple la participation des deux époux à la prise de décision sur la pratique contraceptive ainsi que la détention de ce pouvoir par l'homme et défavoriserait sa détention par la femme.

\section{Conclusion}

Cette étude montre la persistance d'une répartition sexuelle inégale des statuts et des rôles familiaux, économiques et sociaux dans les deux groups ethniques étudiés. Toutefois, on note aussi d'importants changements sous l'influence de l'instruction et de l'urbanisation. Ces influences sont plus perceptibles en société Bëti qu'en société Bamiléké, reflet d'un passage différencié du traditionnel vers le modernisme. Les Bamiléké restent plus "traditionalistes" dans leurs attitudes, notamment en ce qui concerne la participation masculine aux travaux domestiques, la prise de décisions économique, la polygamie, la répres- sion de l'infidélité féminine. Pour tous les deux groupes, la scolarisation, surtout des femmes, favorise la discussion au sein du couple et le partage du pouvoir de décision. Chez les Bamiléké notamment, la réduction de l'écart d'âges entre conjoints est positivement associée à la discussion au sein du couple et l'union monogamique semble réduire l'inégalité entre conjoints en matière de prise de décision. Aussi bien chez les Bamiléké que les Bëti, les femmes semblent curieusement plus conservatrices des valeurs traditionnelles que les hommes. Ce dernier résultat paraît paradoxal eu égard au débat actuel sur les revendications des femmes pour l'égalité entre hommes et femmes. L'on se demanderait si le problème des femmes ne se trouve pas ailleurs (pauvreté par exemple) que dans les contraintes culturelles. Des recherches futures pourront apporter une réponse à cette interrogation.

\section{Bibliographie}

Abega s. c. (1987): L'Esona chez les Bëti, Editions clé, Yaoundé.

Alexandre P. et Binet J. (1958): Le groupe dit Pahouin (Fang - Boulou - Bëti), Presse Universitaire de France, $152 \mathrm{p}$. Amselle J.L. (1985): «Ethnies et espaces: pour une anthropologie topologique", in Amselle J. L. et M'Bokolo E. (dir) : Au cœur de l'ethnie : ethnies, tribalisme et état en Afrique, Ed. La Découverte, Paris, pp. II-48.

Assogba L. (|99|): Statut de la femme : une variable explicative de la dynamique démographique, Organisation Internationale du Travail, Genève, I991.

Bertaux D. (1997): Les récits de vie, perspective ethnosociologique, Nathan, Paris, $128 \mathrm{p}$. 
Bisilliat J. (1987): Women of the third world, work and daily life, London and Toronto: Associated University Presses, $97 \mathrm{p}$.

Bisilliat J. (1992): Relations de genre et développement : Femmes et Sociétés, les Editions ORSTOM, Paris, 326 pages.

Bisilliat J. (1997): "Luttes féministes et développement : une double perspective épistémologique» in Bisilliat J. (dir) : Face aux changements les femmes du Sud, I'Harmattan, pp 21 46.

Bochet de The M.P. (1985): «Rites et associations traditionnelles chez les femmes bëti», in Barbier J.C: Femmes du Cameroun, mères pacifiques, femmes rebelles, Orstom, Karthala, Paris, pp. 245-275 .

Boserup E. (1970): Women's role in economic development, New York, St Martin's Press ; paru en Français en 1983: La femme dans le développement économique, Paris, PUF.

Bozon M. (1995): «Les rapports entre femmes et hommes à la lumière des grandes enquêtes quantitatives", in EPHESIA (dir) : La place des femmes, les enjeux de l'identité et de l'égalité au regard des sciences sociales, Paris, la Découverte, pp 655-668.

Brandon A. et Lloyd C. (I99I): "Women's roles in the maintenance of households ; poverty and gender inequality in Ghana", in : Femmes, Famille et Population, vol.l, Dakar, UEPA, (pp. 109-143) (Conférence de Ouagadougou, Burkina Faso, 24-29 avril 1991).

Charmes J. (1997): «Plus visible mais toujours sous-estimée: l'activité économique des femmes en Afrique ", in Bisilliat J. (dir) : Face aux changements les femmes du Sud,
I'Harmattan, pp 47-60.

Copet-Rouger E. (1985): "Contrôle masculin, exclusivité féminine dans la société patrilinéaire", in Barbier J.C: Femmes du Cameroun, mères pacifiques, femmes rebelles, Orstom, Karthala, Paris, 1985, (pp I53-180).

Friedan B. (1964): La femme mystifiée, Collection Femme, Editions Gonthier S. A., Genève, Tome I 239 P., Tome 2 $212 \mathrm{p}$.

Friedan B. (1982): (1977) : Ma vie a changé : écrits sur le mouvement de libération de la femme, Fayard (France), $400 \mathrm{p}$.

Friedan B. (1982): Femmes, le second souffle, Hachette, Paris, 318 p. (origine, I98I).

Gammage S., Maan E., Shehu D., Sadig O. (1997): Women's role in household decision-making : a case study of Nigeria, Washinton, De, International Center for Research on Women, 1997 July. [5], 38 p. : USAID contract $\mathrm{N}^{\circ}$ HRN 5972-c-00-400I-00. USAID cooperative agreement $\mathrm{N}^{\circ}$ DPE 5972-A-00-0036-00.

Hurault J. (1962): Les structures sociales des Bamiléké, Mouton, Paris, I I3 p.

Hurault J. (1970): «Essai de synthèse du système social des Bamilékées ", Africa, NI, Vol. XI, pp. I-24.

Laburthe-tolra P. (I98I): Les seigneurs de la forêt : Essai sur le passé historique, l'organisation sociale et les normes éthiques des anciens Bëti du Cameroun, Publications de la Sorbonne, 490 p.

Laburthe-Tolra P. ( 1985): « Le Mevungu et les rituels féminins à Minlaba ", in Barbier J.C (dir) : Femmes du Cameroun, mères pacifiques, femmes rebelles, Orstom, Karthala, Paris, 1985, pp 233-244.

Locoh T. (1996): "Changements de rôles masculins et féminins dans la crise : la révolution silencieuse" in Coussy J. et 
Vallin J. (dir.) : Crise et Population en Afrique, les Etudes du CEPED, $n^{\circ} 13$, pp. 445-469.

Locoh T. (1997): «Programmes de populations et amélioration du statut des femmes, une convergence difficile ", in Bisilliat J. (dir) : Face aux changements les femmes du Sud, l'Harmattan, pp 285-296.

Locoh T., Labourie-racape A. et Tichit C. (1996): Genre et développement : des pistes à suivre, Documents et Manuels du CEPED n ${ }^{\circ} 5$, Paris, I 54 pages.

Locoh T. et Labourie-Racape A. (1997): "Genre et démographie : nouvelles problématiques ou effet de mode?», communication présentée à la Chaire Quetelet 1997, Louvain-la-neuve, novembre 1997, $24 \mathrm{p}$.

Mathieu N.C. (1973): Homme-culture et femme-nature?, L'Homme, juilletseptembre 1973, XIII(3).

Mbock C.G. (1984): «Parole, femme et pouvoir traditionnels dans les sociétés segmentaires Basaa et Bëti du SudCameroun», MESRES, revue Science et Technique, Serie Sciences Humaines, Vol. 2, No I-2, Yaoundé, (PP 9I-106).

Ngoa H. (1968): Le mariage chez les Ewondo, thèse de doctorat de troisième cycle, Université de Paris, Faculté des lettres et Sciences Humaines (Sorbonne), $270 \mathrm{p}$.

Oppong C. (1988): "Les femmes en
Afrique : des épouses, des mères et des travailleuses" in Tabutin D. Population et sociétés en Afrique au Sud du Sahara, Harmattan, Paris, 1988 (pp 42I-440).

Stamp P. (1989): La technologie, le rôle des sexes et le pouvoir en Afrique, CRDI, 213 pages.

Tardits C. (1960): Contribution à l'étude des populations Bamiléké de l'Ouest Cameroun, Ed Berger-Levrault, Paris, $133 \mathrm{p}$.

Tardits C. (198I): Contribution de la recherche ethnologique à l'histoire des civilisations du Cameroun, Paris, CNRS, vol I et 2, $597 \mathrm{p}$.

Tchouanga T.P. et Ngangoum B.F. (1975): La vérité du culte des ancêtres en Afrique chez les Bamiléké, Ed. Essor des Jeunes, Nkongsamba, $80 \mathrm{p}$.

Veron J. (1997): Le monde des femmes : inégalité des sexes, inégalité des sociétés, SEUIL, Paris, 206 p.

Walker K. (1997): " Pour la reconnaissance sociale des tâches domestiques des femmes » in Michel A. (dir) (1997): Femmes, sexisme et sociétés, Presses Universitaires de France, Paris, pp. 17I-I77.

Yana S.D. (1997): «Entre subordination et pouvoir : statuts et rôles féminins au Cameroun : réalités d'hier, images d'aujourd'hui», in Politique africaine: l'Afrique des femmes, $10 \mathrm{p}$. 


\section{Annexe}

Tableau AI La discussion et la prise de décision au sein du couple selon le niveau d'instruction du couple et l'ethnie (EDS, 1998)

\begin{tabular}{|c|c|c|c|c|c|c|c|c|c|c|}
\hline Discussion et prise de & & & amiléke & & & & & Bêti & & \\
\hline $\begin{array}{l}\text { Discussion au sein du } \\
\text { couple } \\
\text { sur la PF }\end{array}$ & $\begin{array}{l}\text { Homo } \\
\text { élevé } \\
(*)\end{array}$ & $\begin{array}{l}\text { Hypo } \\
\text { moyen }\end{array}$ & $\begin{array}{l}\text { Hyper } \\
\text { moyen }\end{array}$ & $\begin{array}{l}\text { Homo } \\
\text { faible }\end{array}$ & Ens & $\begin{array}{l}\text { Homo } \\
\text { élevé } \\
(*)\end{array}$ & $\begin{array}{l}\text { Hypo } \\
\text { moyen }\end{array}$ & $\begin{array}{l}\text { Hyper } \\
\text { moyen }\end{array}$ & $\begin{array}{l}\text { Homo } \\
\text { faible }\end{array}$ & Ens \\
\hline$\overline{\text { Oui }}$ & 56,2 & 55,8 & 37,0 & 38,6 & 47,0 & 68,1 & 55,4 & 45,4 & 44,8 & 55,2 \\
\hline $\begin{array}{l}\text { Non } \\
\text { (Effectif) }\end{array}$ & $\begin{array}{l}43,8 \\
(194)\end{array}$ & $\begin{array}{l}44,2 \\
(52)\end{array}$ & $\begin{array}{l}63,0 \\
(81)\end{array}$ & $\begin{array}{l}61,4 \\
(17 I)\end{array}$ & $\begin{array}{l}53,0 \\
(498)\end{array}$ & $\begin{array}{l}31,9 \\
(166)\end{array}$ & $\begin{array}{l}44,6 \\
(56)\end{array}$ & $\begin{array}{l}54,6 \\
(99)\end{array}$ & $\begin{array}{l}55,2 \\
(116)\end{array}$ & $\begin{array}{l}44,8 \\
(437)\end{array}$ \\
\hline $\begin{array}{l}\text { Prise de décision sur la } \\
\text { pratique contraceptive }\end{array}$ & $(*)$ & & & & & $(*)$ & & & & \\
\hline L'homme & 38,0 & 35 & & 25,2 & 33,6 & 40,0 & & 5,4 & 39,1 & 45,1 \\
\hline La femme & 37,4 & 49 & & 54,1 & 45,5 & 45,5 & & 4,8 & 34,8 & 39,6 \\
\hline $\begin{array}{l}\text { Les deux } \\
\text { (Effectif) }\end{array}$ & $\begin{array}{l}24,6 \\
(17 I)\end{array}$ & $\begin{array}{l}15 \\
(10\end{array}$ & & $\begin{array}{l}20,7 \\
(1 I I)\end{array}$ & $\begin{array}{l}20,9 \\
(387)\end{array}$ & $\begin{array}{r}14,5 \\
(145)\end{array}$ & $\begin{array}{r}9 \\
(1\end{array}$ & $\begin{array}{l}8 \\
12)\end{array}$ & $\begin{array}{l}26,1 \\
(69)\end{array}$ & $\begin{array}{r}15,3 \\
(326)\end{array}$ \\
\hline
\end{tabular}

Notes : I) idem tableau I ; 2 ) Instruction du couple : Homo élevé = les deux conjoints ont un niveau secondaire ou plus ; Hypo moyen = la femme a un niveau secondaire ou plus et l'homme est non scolarisé ou a un niveau primaire ; Hyper moyen = l'homme a un niveau secondaire ou plus et la femme est non scolarisée ou a un niveau primaire ; Homo faible $=$ les deux conjoints sont non scolarisés ou ont un niveau primaire

Tableau A2 Discussion et prise de décision au sein du couple selon l'activité économique du couple et l'ethnie (EDS, 1998)

\begin{tabular}{|c|c|c|c|c|c|c|c|c|c|}
\hline \multirow{2}{*}{$\begin{array}{l}\text { Discussion et prise de } \\
\text { décision au sein du } \\
\text { couple }\end{array}$} & \multicolumn{5}{|c|}{ Bamiléke } & \multicolumn{4}{|c|}{ Bêti } \\
\hline & $\begin{array}{l}\text { Homo } \\
\text { élevé }\end{array}$ & $\begin{array}{l}\text { Hypo } \\
\text { moyen }\end{array}$ & $\begin{array}{l}\text { Hyper } \\
\text { moyen }\end{array}$ & $\begin{array}{l}\text { Homo } \\
\text { faible }\end{array}$ & Ens & $\begin{array}{l}\text { Homo } \\
\text { élevé }\end{array}$ & $\begin{array}{l}\text { Hypo } \\
\text { moyen }\end{array}$ & $\begin{array}{l}\text { Hyper } \\
\text { moyen }\end{array}$ & $\begin{array}{l}\text { Homo Ens } \\
\text { faible }\end{array}$ \\
\hline
\end{tabular}

Discussion au sein du (*)

couple sur la PF

$(*)$

$\begin{array}{lllllllllll}\text { Oui } & 55,8 & 52,0 & 52,3 & 41,9 & 49,1 & 67,3 & 58,8 & 65,3 & 50,2 & 56,0 \\ \begin{array}{l}\text { Non } \\ \text { (Effectif) }\end{array} & 44,2 & 48,0 & 47,7 & 58,1 & 50,9 & 32,7 & 41,2 & 34,7 & 49,8 & 44,0 \\ & & & & & & & & & & \\ & (113) & (98) & (153) & (210) & (574) & (55) & (51) & (98) & (287) & (491)\end{array}$

Prise de décision sur (-)

(*)

la pratique

(-) $\quad(*)$

contraceptive

$\begin{array}{lcccccccc}\text { L'homme } & 29,9 & 32,5 & 33,3 & 32,2 & 31,1 & 39,8 & 46,4 & 42,4 \\ \text { La femme } & 40,2 & 45,6 & 46,3 & 40,7 & 42,2 & 49,6 & 36,2 & 41,3 \\ \text { Les deux } & 29,9 & 21,9 & 20,4 & 23,1 & 26,7 & 10,6 & 17,4 & 16,3 \\ \text { (Effectif) } & (97) & (206) & (147) & (450) & (45) & (123) & (207) & (375)\end{array}$

Notes : I) idem tableau 3.I;

2) Activité économique du couple : homo élevé = les deux conjoints ont le même pouvoir économique élevé (c'està-dire exercent dans le commerce, les professions libérales ou sont cadres supérieurs ou techniciens des administrations publique ou privée); homo faible = les deux conjoints ont le même pouvoir économique faible (c'està-dire sont agriculteurs, manœuvres ou exercent dans les activités autres); hypo moyen = la femme a un pouvoir économique supérieur à celui de l'homme; hyper moyen = l'homme a un pouvoir économique supérieur à celui de la femme. 
Tableau A3 Discussion et prise de décision au sein du couple selon l'écart d'âges entre conjoints et l'ethnie (EDS, 1998)

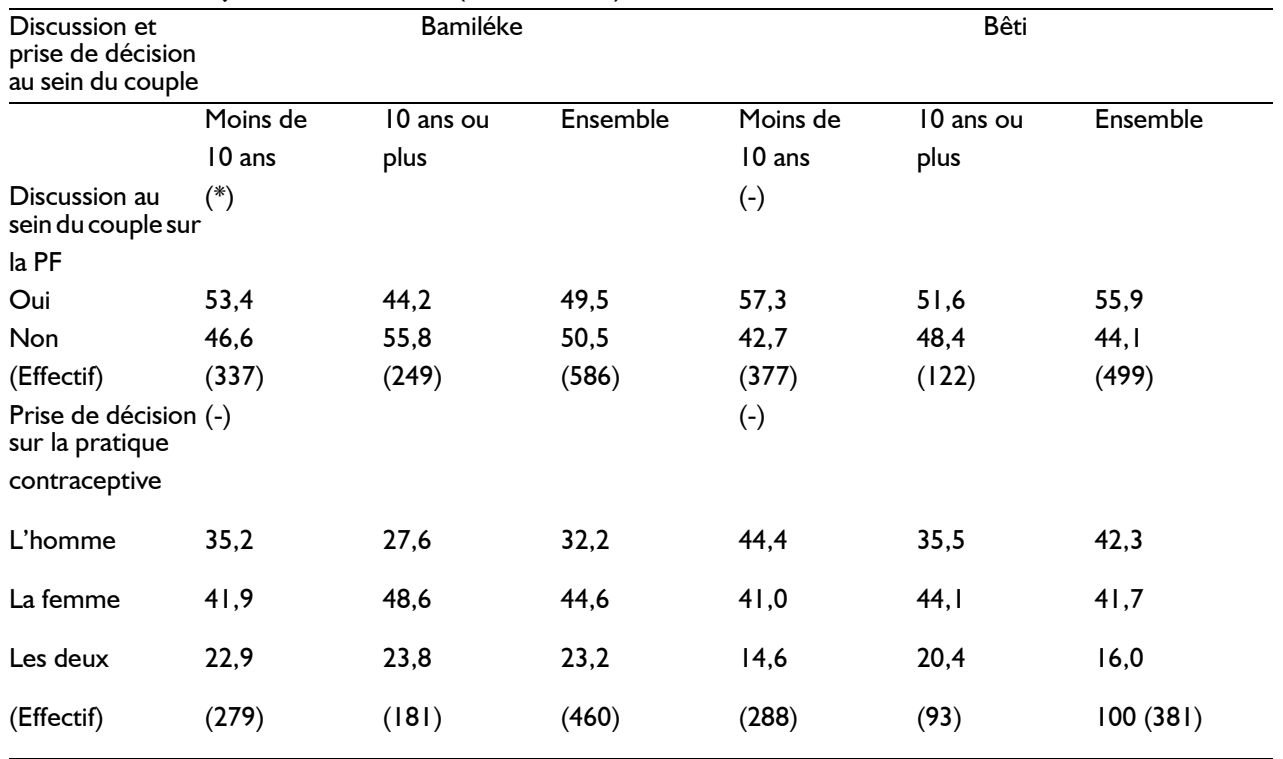

Notes : I) idem Tableau I

Tableau A4 La discussion et la prise de décision au sein selon le type d'union et l'ethnie (EDS, 1998)

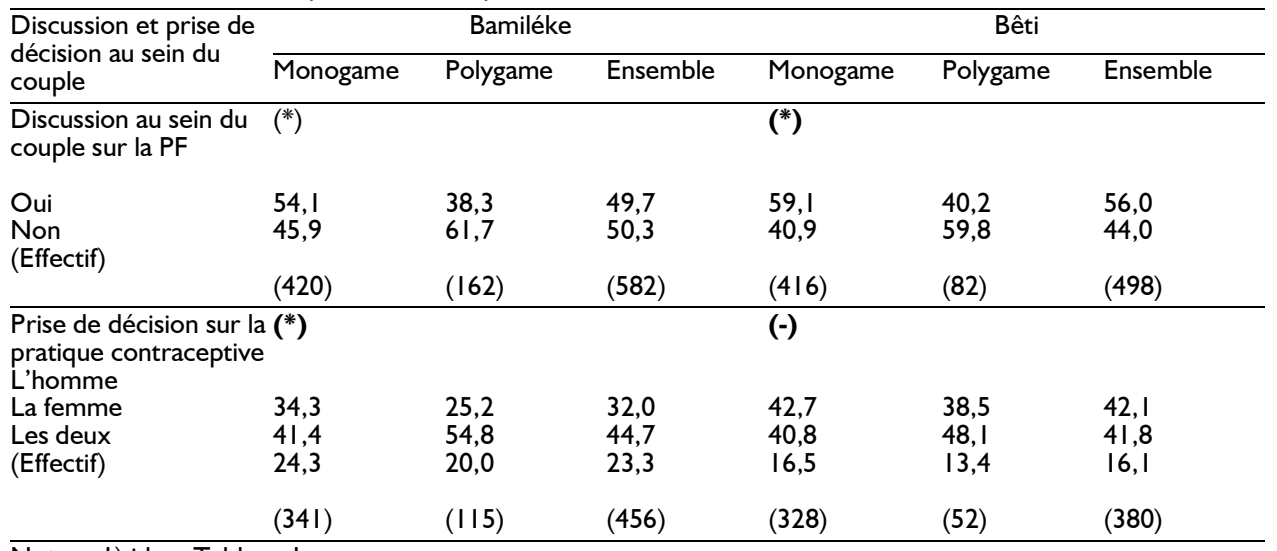

Notes : I) idem Tableau I 